\title{
Impacto del practicum en las creencias de los maestros en formación sobre la relación familia-escuela
}

\author{
ANA CASTRO ZUBIZARRETA \\ Universidad de Cantabria, Cantabria, España \\ MARÍA ROSA GARCÍA-RUIZ \\ Universidad de Cantabria, Cantabria, España
}

PABLO MARAVER LÓPEZ

Universidad de Huelva, Huelva, España

\section{RESUMEN}

El pensamiento del profesorado está influenciado por su formación inicial, sus características personales y el contexto en el que se desenvuelve, siendo su experiencia práctica crucial para que determinadas creencias se modifiquen al configurar su identidad profesional docente. Se analiza el impacto que el practicum puede tener en la formación de los maestros respecto a sus creencias sobre la relación familia-escuela. Para ello se aplicó un cuestionario a 203 maestros españoles en formación del grado de educación infantil y primaria. Los resultados muestran que existen cambios sustanciales en su pensamiento tras su experiencia en los centros educativos. Sus creencias sobre la importancia de la relación familia-escuela se refuerzan, así como la importancia de la acción tutorial y se valoran significativamente los nuevos canales de comunicación. Las conclusiones enfatizan la necesidad de generar una actitud crítica y reflexiva en el profesorado desde su formación inicial.

PALABRAS CLAVE

familia; educación; creencias; participación; maestros. 


\section{PRACTICUM IMPACT ON THE BELIEFS OF PRESERVICE TEACHERS ABOUT FAMILY-SCHOOL RELATIONSHIP}

\section{ABSTRACT}

The teachers thought is influenced by their initial training, personal characteristics and the context in which it operates, and it is crucial to certain beliefs to be modified when configuring their teaching professional identity. Impact that practicum elaboration can have regarding their beliefs about the family-school relationship is analyzed. A questionnaire was applied to 203 Spanish students in the grade of teacher training of primary and early childhood education. The results show that there are substantial changes in the way of thinking after their experience in schools. Beliefs about the importance of family-school relationship are reinforced, so as the importance they concede to the guidance and tutorial action and the new channels of communication present between families and school for interacting are significantly valued. Conclusions emphasize the need to generate a critical attitude towards the teachers from their first contact with teaching.

KEYWORDS

family; education; beliefs; participation; teachers.

\section{IMPACTO DO ESTÁGIO EM CRENÇAS DE PROFESSORES EM FORMAÇÃO NA RELAÇÃO FAMÍLIA-ESCOLA}

RESUMO

O pensamento dos professores é influenciado pela formação inicial, por características pessoais e pelo contexto em que opera, sendo a sua experiência prática crucial para que certas crenças sejam modificadas ao configurar sua identidade profissional docente. $\mathrm{O}$ impacto que o estágio pode ter na formação dos professores no que diz respeito às suas crenças sobre a relação família-escola é analisado. Para esse fim, um questionário foi aplicado a 203 estudantes espanhóis na fase de estágio profissional na formação de professores da educação infantil e ensino primário. Os resultados mostram que há mudanças substanciais na maneira de pensar após a experiência em escolas. Crenças sobre a importância da relação família-escola são reforçadas, bem como a importância da orientação e da ação tutorial, com os novos canais de comunicação presentes entre famílias e escola para interação sendo significativamente valorizados. As conclusões enfatizam a necessidade de se promover uma atitude crítica e reflexiva por parte dos professores em sua formação inicial.

PALAVRAS-CHAVE

família; educação; crenças; participação professores. 


\section{INTRODUCCIÓN}

Pese al notable consenso existente en la investigación educativa sobre los beneficios que se desprenden de establecer vínculos fuertes y honestos entre los miembros de la comunidad escolar (Epstein, 1995), la realidad actual evidencia que la relación familia-escuela sigue siendo un reto atemporal para nuestros centros educativos.

Aunque ambas agencias educativas deben considerarse binomios inseparables, con frecuencia, se percibe la distancia, el desencanto y el conflicto entre ambas. A pesar de ello, señala Garreta (2015) que familias y escuelas se necesitan y precisan cuidar y velar por una relación que es entendida como un factor clave en la educación del alumnado y que supone la necesidad de afrontar la responsabilidad de mejorar la relación con el otro.

Así, familia y escuela son representadas en la literatura científica como dos escenarios sociales diferenciados y peculiares, con dificultades en sus relaciones, pero también con posibilidades de encuentro; con necesidades, intereses y funciones propias, pero que tienen un protagonista en común, el niño (López, Ridao y Sánchez, 2004), y que por su bien, han de establecer relaciones bidireccionales basadas en el intercambio de información, en el respeto mutuo y en la confianza (De Oliveira e Silva, 2014), aspecto en el que insiste este autora, como elemento constitutivo de esta relación. Reforzar la confianza de las familias en la institución escolar es por tanto un asunto primordial en el que es preciso seguir trabajando, tan importante como la confianza de los docentes en las familias, aspecto que debe pasar por un conocimiento en profundidad por ambas partes.

Desde hace décadas se viene relatando que la implicación y participación de las familias en la escuela incrementa los resultados escolares del alumnado, su desarrollo personal y social (Brookmeyer, Fanti y Henrich, 2006; Cross y Barnes, 2014; Epstein, 2001), mejora la competencia autopercibida de las familias, incide en el desarrollo de una parentalidad positiva (García-Bacete, 2003; Olmsted, 1991) y favorece que la escuela se convierta en un contexto más dinámico, participativo y democrático (Batanova y Loukas, 2014; Furman 2004), en el que, por lo tanto, las relaciones entre los diferentes miembros de la comunidad educativa tienen un valor indiscutible en diferentes ámbitos. Dichas relaciones son sustancialmente singulares en función del tipo de participación que se establezca, y que según los autores ya citados pueden definirse en tres modalidades singulares y complementarias en su desarrollo:

Participación informativa o pseudoparticipación, en el caso de que las familias asumen su relación con la escuela como meras receptoras de información que tiene que ver con el proyecto educativo del centro y con el proceso de enseñanza y aprendizaje de sus hijos, pero sin poder de decisión, puesto que es el centro, o en su caso el docente quien toma la iniciativa en este tipo de interacción.

Participación consultiva, en la que las familias forman parte de los órganos de gobierno del centro, como miembros de la comunidad educativa, representadas en el Consejo Escolar u otros organismos reconocidos en cada país para representar a la comunidad educativa. En esta modalidad, las familias además de recibir información, tienen poder de decisión a partir de cuestiones planteadas por el centro, tal y como regulan las diferentes normativas al respecto. 
Participación en planes o proyectos del centro, de forma sistemática en el tiempo o bien manera esporádica, en función del tipo de actividad o proyecto planteado por el centro o el docente. En esta modalidad de participación, las familias suelen participar como miembros de las Asociaciones de Madres y Padres de cada centro, en el caso español, por ejemplo, pero también de manera individual a propuesta del equipo educativo, o por iniciativa propia. Esta modalidad de participación es la que permite una mayor vinculación de las familias puesto que garantiza un papel activo, con capacidad de toma de decisiones y con la responsabilidad compartida en cuantas tareas se planeen relacionadas con la educación de sus hijos.

No podemos obviar que la relación familia-escuela, puede desencadenar tanto en interacciones positivas como negativas (Llevot y Bernard, 2015), que pueden derivar en una relación sustentada en la colaboración, o en una relación impuesta que tiende a controlar la invasión o la intrusión del otro en el espacio propio, donde Este, es percibido como un extraño (Arnáiz, 1999; Maulini, 1997). En este sentido, coincidimos con Garreta (2015) cuando señala que un factor clave para la participación es la comunicación, marco de una adecuada relación. Entendemos, por tanto, que la participación se deriva de una interacción positiva que se sustenta en una actitud de colaboración que se fomenta si en la escuela existe un clima que favorezca en sus miembros la percepción de sentirse escuchados, respetados y valorados (Castro y García-Ruiz, 2013). De este modo, "hablar de participación en el ámbito educativo supone la necesaria cooperación y coordinación entre los distintos sectores de las comunidades educativas, especialmente entre familia y profesorado" (Hernández et al., 2016, p. 4).

Asimismo, la participación de las familias es considerada fundamental para el buen funcionamiento del centro educativo (Comisión Europea, 2000), y comprende el ejercicio de diversos roles por parte de las familias (Garreta, 2007; Hester, 1989), como son la comunicación con el profesorado y resto de personal de la escuela, la participación en asociaciones de padres y madres, la participación en actividades escolares, el apoyo en las tareas escolares de los hijos y el ejercicio de su rol como educadores.

Es notable el esfuerzo institucional realizado para optimizar la participación de las familias en la escuela, diseñando un marco normativo que regule la representación de las familias en las decisiones de gestión, reconociendo los derechos de los padres como colectivo. Así, todos los países europeos cuentan con sistemas organizados expresamente para favorecer su participación en las estructuras de gestión, aunque varían de un país a otro (Eurydice, 1997).

Concretamente, en España, los dos procedimientos más utilizados para garantizar la participación de las familias en la escuela son la existencia de asociaciones de madres y padres y su inclusión en los órganos en los que están representados los diferentes sectores que componen la comunidad educativa, ya sea a nivel del centro o a nivel local, regional o nacional. A este respecto, hay que destacar que el tipo de participación o vinculación difiere en función del nivel en el que nos situemos, siendo una participación, en la mayoría de los casos, más directa y con mayor poder e implicación en la toma de decisiones cuanto más cercana al centro de establezca dicha vinculación. 
Los estudios que han abordado el estado de la participación de las familias en la escuela (Feito, 1992; Garreta, 2008; Kñallinsky, 1999; Luengo y Moya, 2008; Santos, 1999) señalan la importancia de la relación familia-escuela, los obstáculos percibidos y las demandas y propuestas para potenciar dicha relación. A pesar de la larga trayectoria de investigaciones sobre este ámbito de estudio, Navaridas y Raya (2012) afirman que la participación dista mucho de ser activa y significativa. Sin embargo, el reciente informe del Consejo Escolar del Estado español (2014) aporta una mirada más positiva a los datos de la participación de las familias en los centros escolares, en la línea de Kñallinsky (1999), quien defiende que, ante la poca participación de las familias, la existencia de la misma, a pesar de ser de baja intensidad, debe ser entendida como positiva.

A pesar de ese matiz positivo, los datos refieren que la participación de las familias en la escuela está limitada a temas marginales o puntuales (Parreira, Walther y Litau, 2013), lo que denota el camino que queda por recorrer para mejorar esta relación e incrementar los niveles de participación familiar.

En este sentido, entre los obstáculos para el encuentro entre familia y escuela descritos por Luengo y Moya (2008) y Garreta (2007, 2008), destacamos la falta de interés o de tiempo, la falta de comprensión o entendimiento mutuo, el desconocimiento del sistema educativo por parte de las familias y la escasa información aportada desde la escuela sobre los mecanismos de participación existentes. Asimismo, consideramos que un obstáculo preocupante es la existencia de resistencias en el profesorado en torno a la participación de la familia, ya que entendemos su figura como clave en la promoción de una adecuada relación. Esta situación es recogida por el informe del Consejo Escolar del Estado (2014) quien advierte sobre la presencia en algunos profesores de la creencia de que las familias y su participación en el centro escolar suponen una interferencia en su trabajo, lo ralentiza o interrumpe. En esta línea se pone de manifiesto otro aspecto limitador derivado de la falta de entendimiento planteado por Garreta (2008), que hace referencia a que los maestros creen que las familias deben implicarse en "su justa medida", pero esa medida está condicionada por un matiz subjetivo que dificulta claramente el acuerdo.

Por otro lado, la tendencia a iniciar el contacto familia-escuela ante la existencia o detección de un problema o dificultad surgida en el niño (Martínez et al., 2000), hace que la relación tome una vertiente correctiva que deja en segundo plano informaciones sobre los avances en el niño, sus grandes conquistas, intereses o preferencias, que originan en las familias temores sobre lo que la escuela va a comunicar. Respecto a los canales de comunicación familia-escuela, los tradicionales son los más utilizados (García et al., 2010), las reuniones de grupo y las entrevistas son las consideradas más útiles para el intercambio de pareceres, siendo necesario prestar mayor atención a los canales emergentes (Webs de centro, blogs de aula, redes sociales...) como dinamizadores de la participación de los progenitores en la escuela, como recomienda el Consejo Escolar del Estado (2014).

Conocer la visión de maestros y familias sobre cómo es su relación resulta imprescindible para poder mejorarla. En este marco de mejora, la formación de los futuros maestros y la detección, análisis y reflexión sobre sus creencias acerca de 
la relación familia-escuela durante su proceso formativo inicial continúan siendo esenciales para la transformación de la realidad actual.

\section{CREENCIAS DE LOS MAESTROS EN FORMACIÓN: UN PILAR EN EL QUE SE ASENTARÁN LAS FUTURAS PRÁCTICAS DOCENTES}

La profesión docente es un proceso que se ve condicionado por el conjunto de experiencias formales y no formales que acontecen a lo largo de la vida. En este sentido, a lo largo de este proceso se van configurando una serie de teorías subjetivas que definen el ejercicio profesional, tal y como lo plantean Martínez y Ahumada (2016). Estos autores ponen de manifiesto una serie de factores que acontecen durante la formación inicial de los docentes y que impactan en la formación profesional, como son el curriculum formal durante su formación universitaria, el método de enseñanza, las características de sus propios profesores durante esta formación inicial, el curriculum oculto, u otros factores como son el tipo de conocimiento impartido en su formación inicial o el nivel de exigencia en la enseñanza.

A lo largo de esta formación inicial y de las experiencias previas al ejercicio profesional, el docente va conformando una serie de creencias que repercutirán ineludiblemente en su práctica profesional. Entendemos por creencias las teorías acerca de sucesos y personas que condicionan la práctica (De la Cruz et al. 2006), premisas o suposiciones sobre algo (Tirado y Aguaded,2014), que orientan la toma de decisiones y las conductas de los sujetos (Prieto, 2008). El estudio de las creencias ayuda a conocer cómo las personas interpretan una realidad, la comprenden, sienten y actúan (Gergen, 1996).

Mucho se ha escrito sobre las creencias del profesorado en su desempeño docente (Argos, 2000; Marrero, 2009; Pajares, 1992), así como sobre las creencias y atribuciones que las familias tienen con relación a la educación de sus hijos (Alcalay, Milicic y Torretti, 2005). Sin embargo, se ha recogido en menor medida el pensamiento del docente sobre los vínculos que a su parecer deben fraguarse con las familias y más aún sobre las creencias que el profesorado en formación posee sobre cómo debería ser la interacción del profesorado con las familias y, por lo tanto, su futura relación con estos en su labor educadora. Gorski (2009) y Amatea (2009) insisten en señalar que la mejora de las relaciones familia-escuela debe encontrar su base en la formación inicial del profesorado, desde la cual se haga consciente al futuro maestro de sus creencias sobre la interacción y relación con las familias de su alumnado. En esta línea, Pozo (2006) plantea que para afrontar procesos de mejora y transformación escolar es necesario cambiar las concepciones o creencias que los agentes implicados tienen sobre la realidad que viven.

Coincidiendo con estos planteamientos, abordamos en esta investigación la influencia del practicum de enseñanza o prácticas profesionales educativas durante la formación de los maestros en el cambio o la consolidación de las creencias sobre la relación familia-escuela, entendiendo que el practicum supone un punto de inflexión para la configuración del pensamiento docente y la construcción de la identidad profesional. 


\section{EL PRACTICUM COMO ELEMENTO ESENCIAL EN LA EXPLORACIÓN DEL PENSAMIENTO DOCENTE}

El practicum se erige como un elemento clave en el que afloran o actúan creencias, no siempre conscientes, que condicionan el desempeño docente y a las que es necesario atender para mejorar la formación inicial del maestro y cambiar así aquellas creencias erróneas iniciales (Chan y Elliott, 2004a; Latorre, 2007; Tigchelaar y Korthagen, 2004). Así, entendemos que el practicum es un observatorio privilegiado de la realidad educativa, que permite al alumnado afianzar creencias o modificarlas en base a la experiencia vivida, teniendo un grado de repercusión significativo en su formación. Algunos autores ponen de relieve que existe una desconexión entre el conocimiento teórico que se adquiere en la universidad y lo que un maestro debe conocer para llevar a cabo la práctica educativa (Fernández-Río y Méndez-Giménez,2013). No obstante, es preciso tener en cuenta que la configuración de estas prácticas profesionales de carácter obligatoria para los maestros en formación difiere entre los diferentes países, o incluso, dentro de un mismo país, entre las diferentes universidades.

Por ello, el practicum no puede desvincularse de la actividad de las aulas universitarias sino que debe ser un recurso para las materias al que acudir para incitar al futuro maestro a reflexionar, evaluar y aprender sobre su enseñanza (Marcelo, 2009), y es precisamente esta capacidad crítica la que pone de relieve Dias (2012) y con la que coincidimos plenamente, puesto que se trata de un valor fundamental que favorece la reflexión sobre la práctica y la intervención posterior en la realidad educativa.

Así, los objetivos que orientan este estudio son los siguientes:

- estudiar el impacto que el practicum o prácticas profesionales educativas puede tener en la formación de los futuros maestros respecto a sus creencias sobre la relación familia-escuela;

- comprobar si el impacto en sus creencias es diferente en función de la especialidad que eligen los futuros maestros (educación primaria o educación infantil).

\section{MÉTODO}

\section{PARTICIPANTES}

La muestra, de carácter aleatorio, estuvo formada por un total de 203 participantes, siendo 108 de la especialidad de educación infantil y 95 de la especialidad de educación primaria siguiendo el siguiente reparto territorial: $21 \%$ estudiantes de la Universidad de Cantabria; 19\% estudiantes de la Universidad de Huelva; 21\% estudiantes de la Universidad de Valladolid; 22\% estudiantes de la Universidad de Valencia; y 17\% estudiantes de la Universidad de Murcia. Del total de participantes el $81 \%$ son mujeres y el $19 \%$ varones. Esta muestra no tiene un carácter representativo, pero sí recoge información generalizada de diferentes regiones españolas, cuyos datos pueden acercarnos a una realidad diversa de los maestros en formación españoles. 
Los maestros graduados en la especialidad de educación infantil en España están habilitados solo para impartir docencia en esta etapa, que abarca desde que los 0 a los 6 años, y que se estructura en dos ciclos educativos, con tres cursos cada uno. Los maestros graduados en la especialidad de educación primaria están habilitados solo para ejercer profesionales en esta etapa, que abarca desde los 6 a los 12 años, estructurada según la ley educativa vigente en seis cursos.

\section{INSTRUMENTO}

Esta investigación sigue un diseño cuasi-experimental con comparaciones pre y post test. El instrumento de recogida de información es un cuestionario tipo Likert con 4 opciones de respuesta según el grado de acuerdo con las variables o afirmaciones planteadas siendo 1 "en desacuerdo" y 4 "totalmente de acuerdo". El cuestionario está formado por 25 ítems y se estructura en tres dimensiones:

1. creencias sobre la participación e implicación de las familias;

2. creencias sobre la acción tutorial y la función docente;

3. cauces de relación familia-escuela.

El análisis de validez de contenido se llevó a cabo tras la aplicación a una muestra piloto de 28 maestros en formación y la aplicación de la valoración por parte de un grupo formado por cinco expertos. La fiabilidad se calculó mediante el alfa de Cronbach, con un resultado de 0,89 y los datos fueron tratados con el programa de análisis estadístico SPSS v19.

\section{PROCEDIMIENTO}

La aplicación del cuestionario se desarrolló mediante una encuesta online, a la que los estudiantes pudieron responder voluntariamente, en primer lugar, antes de realizar el periodo de prácticas y una vez más después de finalizar dicha actividad docente. Conviene matizar que el período de prácticas difiere en cuanto a duración entre las distintas universidades, pero que, en todos los casos analizados en esta investigación, superó los dos meses de duración.

\section{RESULTADOS}

Este apartado presenta un análisis de los resultados obtenidos para conocer la repercusión del practicum o prácticas profesionales educativas en las creencias que los maestros en formación poseen sobre la relación familia-escuela, antes y después de su primera experiencia con la realidad escolar.

Para obtener información acerca de las variables que influyen en estas creencias, se presentan tres niveles de análisis: por dimensiones, por especialidad y por ítems. Así, en la Tabla 1 se presentan los resultados de la dimensión "Creencias sobre la participación e implicación de las familias", en la Tabla 2 la dimensión "Creencias sobre la acción tutorial y la función docente" y en la Tabla 3 la dimensión "Cauces de la relación familia-escuela". También se presenta un análisis correlacional de las mediciones de cada dimensión. En segundo lugar, se analizan 
las diferencias entre la muestra que se corresponde con estudiantes del grado de educación infantil y el grado de educación primaria. Finalmente, se atiende a la especificidad de cada ítem.

\section{Tabla 1 - Creencias sobre la participación e implicación de las familias}

\begin{tabular}{l|l|c|c}
\hline Número & \multicolumn{1}{|c|}{ Creencias } & PRE & POST \\
\hline 2 & $\begin{array}{l}\text { Considero importante que la familia participe de una forma más } \\
\text { continuada en el tiempo (participación sistemática) }\end{array}$ & 3,27 & 3,66 \\
\hline 24 & Las familias tienen mucho que aportar al aula & 3,39 & 3,60 \\
\hline 6 & $\begin{array}{l}\text { Las familias pueden participar en el desarrollo de las actividades del } \\
\text { aula, aportando ideas, información, recursos... }\end{array}$ & 3,19 & 3,52 \\
\hline 7 & Es importante que las familias puedan entrar en el aula & 3,05 & 3,29 \\
\hline 1 & Considero importante la participación de la familia de forma esporádica & 2,55 & 2,83 \\
\hline 20 & $\begin{array}{l}\text { En ocasiones las familias consideran que solo se reclama su } \\
\text { participación para tareas que no aportan mucho a la mejora del } \\
\text { aprendizaje de sus hijos (fiestas, disfraces, salidas...) }\end{array}$ & 2,95 & 2,71 \\
\hline 25 & Las familias pueden entorpecer el ritmo de las clases & 2,01 & 1,82 \\
\hline & Total & 2,91 & 3,06 \\
\hline
\end{tabular}

Fuente: Base de datos de la investigación.

Elaboración propia.

Tabla 2 - Creencias sobre la acción tutorial y la función docente

\begin{tabular}{l|l|c|c}
\hline Número & \multicolumn{1}{|c|}{ Creencias } & PRE & POST \\
\hline 3 & Es función de la tutora fomentar y dinamizar la participación de las familias & 2,98 & 3,31 \\
\hline 4 & Las familias pueden tomar la iniciativa para participar en la escuela & 2,87 & 3,25 \\
\hline 5 & $\begin{array}{l}\text { Se debe mantener contacto y comunicación con otros adultos } \\
\text { responsables del niño que participan en la escuela }\end{array}$ & 3,28 & 3,58 \\
\hline 8 & Es importante que las familias valoren la labor de los tutores & 3,62 & 3,77 \\
\hline 9 & Los tutores deben ser mediadores y resolver conflictos & 3,42 & 3,62 \\
\hline 10 & Las familias deben implicarse más en la educación de sus hijos & 3,60 & 3,81 \\
\hline 17 & $\begin{array}{l}\text { La comunicación con las familias se origina frecuentemente a partir } \\
\text { de un problema en el desarrollo del niño }\end{array}$ & 2,77 & 2,68 \\
\hline 18 & $\begin{array}{l}\text { La comunicación con las familias se origina a partir de un conflicto } \\
\text { entre alumnos }\end{array}$ & 2,51 & 2,48 \\
\hline 19 & $\begin{array}{l}\text { Es importante que el tutor/a explique la metodología que sigue en } \\
\text { el aula con los alumnos a las familias, su forma de trabajo, lo que } \\
\text { considera importante... }\end{array}$ & 3,50 & 3,74 \\
\hline 22 & $\begin{array}{l}\text { Trabajar contenidos que puedan ser generalizables en casa facilita la } \\
\text { participación de las familias }\end{array}$ & 3,32 & 3,61 \\
\hline 23 & En ocasiones las familias no escuchan las orientaciones de la maestra & 2,86 & 3,12 \\
\hline & Total & 3,11 & 3,32 \\
\hline
\end{tabular}

Fuente: Base de datos de la investigación.

Elaboración Propia. 


\section{ANÁLISIS POR DIMENSIONES}

\section{CREENCIAS SOBRE LA PARTICIPACIÓN E IMPLICACIÓN DE LAS FAMILIAS}

El estudio pone de manifiesto la importancia que conceden los maestros en formación a la participación de las familias tanto de forma esporádica como sistemática en el centro escolar, valorando sus aportaciones y la riqueza de la información que pueden trasladar al maestro. Asimismo, consideran que esta participación en las actividades del aula es beneficiosa. Tal y como se observa en la Tabla 1, estas creencias se refuerzan durante el practicum ya que la puntuación de estos ítems $(2,24,6,7$ y 1$)$ se incrementa después del periodo de prácticas.

Del mismo modo, los estudiantes se inclinan más hacia una participación continuada en el tiempo de las familias, frente a otra más esporádica que se entiende como una participación de baja intensidad, dejándose entrever en sus respuestas la importancia que conceden al rol y a la responsabilidad de las familias en la educación de sus hijos. En este sentido, el hecho de que el ítem 20 en la fase post practicum reciba una menor puntuación, supone que los maestros en formación han percibido que las familias consideran que su participación no solo se reclama para tareas puntuales o temas marginales de menor importancia.

Esta visión positiva de la participación de las familias en la escuela se sigue poniendo de manifiesto en el ítem 25, que refiere que los maestros en formación no consideran que las familias puedan entorpecer el ritmo o la dinámica de las clases, creencia que se refuerza después del practicum.

\section{CREENCIAS SOBRE LA ACCIÓN TUTORIAL Y LA FUNCIÓN DOCENTE}

$\mathrm{E}$ l análisis de las creencias respecto a la acción de los tutores y a su función como docentes indica que los maestros en formación están de acuerdo con que las familias deben implicarse aún más en la educación de sus hijos, valorando la labor de los tutores. Así mismo, consideran importante que los tutores ejerzan como

Tabla 3 - Cauces de la relación familia-escuela

\begin{tabular}{l|l|c|c}
\hline Número & \multicolumn{1}{|c|}{ Creencias } & PRE & POST \\
\hline 13 & $\begin{array}{l}\text { Considero que la comunicación con las familias debe realizarse sólo } \\
\text { de forma individualizada }\end{array}$ & 2,08 & 2,14 \\
\hline 14 & $\begin{array}{l}\text { La comunicación con las familias debe realizarse también en } \\
\text { reuniones grupales }\end{array}$ & 3,10 & 3,28 \\
\hline 15 & $\begin{array}{l}\text { Es importante que la comunicación con las familias se realice de } \\
\text { forma oral }\end{array}$ & 3,17 & 3,37 \\
\hline 16 & $\begin{array}{l}\text { La comunicación con las familias también se debe desarrollar de otras } \\
\text { formas: blog, correo electrónico, teléfono }\end{array}$ & 3,04 & 3,32 \\
\hline 21 & $\begin{array}{l}\text { La comunicación con las familias incluye conocer los recursos } \\
\text { materiales con los que se trabaja en el aula }\end{array}$ & 3,05 & 3,49 \\
\hline & Total & 2,89 & 3,12 \\
\hline
\end{tabular}

Fuente: Base de datos de la investigación.

Elaboración propia. 
mediadores en la resolución de conflictos y que puedan comunicar a las familias todo lo relacionado con la metodología implementada en el proceso de enseñanza aprendizaje, favoreciendo la participación a partir de la selección de contenidos generalizables en el contexto familiar (Tabla 2).

Todas las afirmaciones que valoran las "Creencias sobre la acción tutorial y la función docente" varían en el grado de acuerdo en las dos mediciones y van de menor a mayor grado de acuerdo una vez vivida su primera experiencia práctica en el centro educativo. Como excepción encontramos las creencias que hacen referencia a que la comunicación con la familia se origina a partir de un conflicto o problema en el desarrollo del niño o a partir de un conflicto surgido entre los alumnos (ítems 17 y 18). Esta disminución en el grado de acuerdo con esas dos creencias tiene una interpretación positiva ya que la experiencia práctica modifica estas creencias sobre una comunicación con las familias centrada principalmente en planteamientos correctivos.

\section{CAUCES DE LA RELACIÓN FAMILIA-ESCUELA}

$\mathrm{E}$ l análisis de resultados relacionados con los cauces o la forma de comunicarse la familia con los docentes indica que los maestros en formación consideran la importancia de utilizar un abanico amplio de canales de comunicación que favorezcan la relación. Así se pone de relieve en los datos que se desprenden del ítem 13, donde los maestros en formación señalan que la comunicación con las familias no debe realizarse únicamente de forma individualizada. En esta línea, apuntan a la importancia de las reuniones grupales, y se decantan por la comunicación oral para enmarcar la relación familia-escuela. Una relación, que puede ser dinamizada con el uso de otros medios emergentes de comunicación (ítem 16). Al respecto, los resultados obtenidos en este ítem muestran cómo los maestros en formación consideran importante ampliar los canales de comunicación tradicionales usados por excelencia en los centros escolares, creencia que es reforzada después del periodo de prácticas.

El mayor grado de acuerdo de los maestros en formación en relación a esta dimensión de análisis se encuentra en torno a la importancia que le conceden a que las familias conozcan los recursos materiales con los que se trabaja en el aula (ítem 21), grado de acuerdo que se relaciona con las respuestas obtenidas en el ítem 19 comentado en el epígrafe anterior y que muestra cómo los estudiantes consideran necesario trasladar a las familias informaciones sobre la dinámica de las clases, metodologías, criterios de trabajo, etc., que ayuden a comprender a los progenitores lo que acontece en el contexto escolar y así construir unas bases para el entendimiento y el trabajo colaborativo (Tabla 3).

Todas las creencias valoradas en esta dimensión se refuerzan después de las prácticas de enseñanza al incrementar en todos los ítems su puntuación.

\section{COMPARACIÓN ENTRE LAS MEDICIONES PRE Y POST DE}

\section{CREENCIAS DEL PROFESORADO ANTES Y DESPUÉS DEL PRACTICUM}

La prueba $T$ para muestras relacionadas fue realizada para evaluar si existe una diferencia estadísticamente significativa entre las medias de las puntuaciones anteriores y posteriores al periodo de experiencia práctica en la escuela. Los resultados fueron significativos, $\mathrm{t}(193)=9.718, \mathrm{p}<.0005$, indicando que hay un incremento entre la medida pre $(M=2,99, S D=0,023, N=194)$ y post $(M=3,20, S D=0,022)$. 
$\mathrm{E} 1$ incremento de la media fue 0,201 , con un intervalo de confianza del $95 \%$ para la diferencia entre las medias de 0,16 y 0,24. Por lo tanto hay una diferencia significativa después de la primera experiencia práctica en la escuela. Estos datos nos señalan la relevancia y el impacto del practicum de enseñanza en la formación de los futuros maestros respecto a la relación familia-escuela.

En la primera dimensión se obtiene una correlación de 0,475 entre sus mediciones pre y post. En la dimensión II se obtiene una correlación de 0,615 y en la dimensión III se obtiene una correlación de 0,548 . Todo ello nos indica que, aunque la correlación es positiva en todos los casos, no existe una alta correlación entre la medición previa a las prácticas con la medición posterior. Es decir, una puntuación alta en la primera medición no conlleva obligatoriamente otra puntuación alta en la otra. En resumen, las tres dimensiones correlacionan positivamente, ya que se encuentran diferencias estadísticamente significativas entre las puntuaciones en las medidas pre y post efectuadas antes y después de realizar las prácticas en el centro escolar.

\section{ANÁLISIS POR ESPECIALIDAD}

A continuación, se presenta la comparación de creencias entre los maestros en formación de educación infantil y primaria. Para ello se realiza una comparación de medias independientes a través de la prueba $\mathrm{T}$ de Student para comprobar si existen diferencias estadísticamente significativas en las dos especialidades del grado (Tabla 4).

Los datos que se desprenden de la medición antes de realizar el período de prácticas en los centros obtienen diferencias estadísticamente significativas, si bien, en la fase post-practicum, las diferencias de las medias se reducen y no son estadísticamente significativas.

Tabla 4 - Comparativa de creencias entre maestros en formación atendiendo a la especialidad de infantil y primaria

\begin{tabular}{l|c|c|c|c}
\hline & Grupo & N & Mean & Significación \\
\hline \multirow{2}{*}{ DIM_I_PRE } & Infantil & 108 & 3,0265 &, 000 \\
\cline { 2 - 5 } & Primaria & 95 & 2,7458 & \\
\hline \multirow{2}{*}{ DIM_I_POST } & Infantil & 108 & 3,0860 &, 099 \\
\cline { 2 - 5 } & Primaria & 95 & 3,0930 & \\
\hline \multirow{2}{*}{ DIM_II_PRE } & Infantil & 108 & 3,1852 &, 000 \\
\cline { 2 - 5 } & Primaria & 95 & 3,0221 & \\
\hline \multirow{2}{*}{ DIM_II_POST } & Infantil & 108 & 3,3491 &, 136 \\
\cline { 2 - 5 } & Primaria & 95 & 3,2756 &, 001 \\
\hline \multirow{2}{*}{ DIM_III_PRE } & Infantil & 108 & 2,9481 &, 307 \\
\cline { 2 - 5 } & Primaria & 95 & 2,8116 & \\
\hline \multirow{2}{*}{ DIM_III_POST } & Infantil & 108 & 3,1574 & \\
\cline { 2 - 5 } & Primaria & 95 & 3,0767 & \\
\hline
\end{tabular}

Fuente: Base de datos de la investigación.

Elaboración propia. 
La prueba $T$ para muestras independientes arroja que en 21 de las 25 creencias el grado de acuerdo de los estudiantes de educación primaria incrementa por encima de los resultados obtenidos por los estudiantes de la especialidad de infantil con relación a la medida post-practicum. Esto pone de manifiesto la repercusión del practicum en las creencias de los estudiantes, principalmente en la especialidad de educación primaria.

En este sentido, resulta de interés mostrar, a modo de ejemplo, un ítem de cada dimensión, para observar cómo influye la variable especialidad en las dos mediciones. En la dimensión I destaca el ítem 7, "Creo que es importante que las familias puedan entrar en el aula", en el que las puntuaciones post-practicum en primaria muestran un incremento (pre $=2,67$ - post $=3,06$ ) en el grado de acuerdo hacia la participación de las familias en la dinámica del aula, mientras que en infantil sigue siendo alto el grado de acuerdo (pre=3,34- post=3,47), consolidando la importancia que conceden a dicha participación.

Respecto a la dimensión II, el ítem 19, "Pienso que es importante que el(la) tutor/a explique la metodología que sigue en el aula con los alumnos a las familias, su forma de trabajo, lo que considera importante...", presenta un incremento considerable en las mediciones pre-post en la especialidad de educación primaria (pre $=3,26-$ post $=3,65$ ). Por su parte, en Infantil se observa un incremento más leve (pre=3,69post $=3,81$ ). Destaca el elevado grado de acuerdo que conceden ambas especialidades al hecho de que la familia conozca la metodología empleada por el docente.

De la dimensión III, que hace referencia a los canales de comunicación, hemos considerado presentar los resultados del ítem 16 que hace mención del uso de canales de comunicación diferentes a los tradicionales en los que destaca la incidencia del practicum en los estudiantes mostrando nuevamente un incremento en el grado de acuerdo sobre la incorporación de la tecnología como vía de comunicación entre familia y escuela. Los resultados obtenidos en primaria (pre=2,73- post $=3,14$ ) y en la muestra de educación infantil (pre=3,28- post=3,47) mantienen la tendencia hacia un mayor grado de acuerdo.

\section{ANÁLISIS POR ÍTEMS}

Finalmente se analizan las creencias más representativas de cada dimensión. Para la dimensión I, "Creencias sobre la participación e implicación de las familias", se encuentra un alto grado de acuerdo en las consideraciones sobre la importancia de la participación activa y continuada, así como la presencia y aportaciones de las familias en el aula. Así, las creencias como "Considero importante que la familia participe de una forma más continuada en el tiempo (participación sistemática)" y "Considero que las familias tienen mucho que aportar al aula", obtienen una puntuación media de 3,66 y 3,60 puntos respectivamente.

En la dimensión II, "Creencias sobre la acción tutorial y la función docente", destacan aquellas creencias que fomentan el acercamiento entre la metodología y los contenidos que se trabajan en el aula con las actividades que se pueden realizar en casa. Al respecto, "Pienso que es importante que el(la) tutor/a explique la metodología que sigue en el aula con los alumnos a las familias, su forma de trabajo, lo que considera importante..." obtiene una puntuación media de 3,74, mientras que "Considero que trabajar contenidos que puedan ser generalizables en casa facilita la participación de las familias" obtiene 3,61 puntos. 
En la dimensión III, “Cauces de la relación familia-escuela”, se evidencia la necesidad llevar a cabo procesos de comunicación en los que las familias puedan conocer los recursos educativos mediante reuniones grupales, de forma oral o por vías telemáticas superando estas creencias los 3,30 puntos de media.

En general, se encuentran diferencias significativas entre ambas mediciones pre y post, con un intervalo de confianza del $95 \%$ en 16 de las 25 creencias del cuestionario, en su mayoría pertenecientes a las dimensiones "Creencias sobre la acción tutorial y la función docente" y "Cauces de la relación familia-escuela". Por lo tanto, debemos tener en cuenta el carácter definitorio e impactante de las prácticas ya que influyen significativamente en un $74 \%$ de las afirmaciones planteadas sobre la percepción del profesorado en formación respecto a la participación de las familias en la escuela.

Las creencias 13 y 25 , son las que menor puntuación reciben, indicando que el $82 \%$ pre y $74 \%$ post de las personas participantes no está de acuerdo con que la comunicación con la familia se realice sólo de forma individualizada (n.13). Por su parte, para la creencia n. 25, la mayoría (84\%) del profesorado en formación está poco de acuerdo o en desacuerdo con la afirmación que sostiene que las familias pueden entorpecer el ritmo de las clases.

Finalmente, las diferencias en las medias de infantil y primaria son estadísticamente significativas en 9 creencias que en su mayoría corresponden a la dimensión "Creencias sobre la participación e implicación de la familia en la escuela" (n. 1, 7, $11,12,13,20,22$ y 25). Esto indica que en la etapa de educación infantil existe la creencia de que se da una mayor participación e implicación de la familia que en educación primaria.

\section{CONCLUSIONES}

La formación de los maestros de educación infantil y primaria, tanto inicial como continua, ha sido objeto de estudio de numerosas investigaciones (Argos, 2000; Marcelo, 2009), entre las que destacamos aquellas que analizan la importancia del practicum como factor clave de su formación, así como la necesidad de reforzar su pensamiento crítico y reflexivo y que han guiado nuestra investigación (Chan y Elliott, 2004b; Dias, 2012).

La relación familia-escuela constituye un elemento fundamental para la mejora de la calidad educativa, que se da a conocer a los estudiantes desde las universidades. Dicha relación está determinada por las creencias del profesorado que dictan su actuación y práctica pedagógica. Conocer las creencias que los maestros en formación poseen sobre la relación familia-escuela y cómo éstas se modifican, se mantienen o refuerzan tras el primer contacto con el practicum supone la oportunidad de poder reflexionar y acompañar al maestro en formación en la construcción de su identidad y pensamiento docente, pudiendo articular el conocimiento eminentemente teórico transmitido desde las aulas universitarias con el potencial práctico que se experimenta en el practicum.

Asimismo, se identifican creencias que pueden dificultar la relación familia-escuela asentadas en el pensamiento del maestro en formación y a las que es necesario prestar atención. Al respecto, un 16\% de los participantes en el estudio considera que 
las familias entorpecen la acción docente y un 26\%, después del practicum, considera que la relación familia-escuela debe realizarse sólo de forma individualizada. Estos resultados debieran ser foco de reflexión en las aulas universitarias siguiendo la línea planteada por otros estudios de gestar propuestas prácticas que favorezcan la mejora de las relaciones familia-escuela (Bastiani, 1989; Blank y Kershaw, 1998; Boult, 2006; Grant y Ray, 2010), y que desde nuestro parecer debieran incidir en la formación de los futuros maestros ayudando así a asentar las bases de una relación que si bien es compleja (Arnáiz, 1999; Santos,1999), es totalmente necesaria.

Así lo entienden los estudiantes que han participado en este estudio, cuyas creencias apuntan a considerar la relación familia-escuela como un factor fundamental de la educación, tanto antes como especialmente después de su primera experiencia práctica en un centro educativo. En este sentido se constata que, tras el período de prácticas en el centro, las diferentes creencias analizadas en este trabajo se ven reforzadas tanto en los estudiantes de educación primaria como en los de educación infantil. Por tanto, podemos concluir que es relevante el impacto que el practicum tiene con relación a las creencias de los futuros maestros. Además, los resultados revelan cómo el maestro en formación considera necesario ampliar los canales de comunicación existentes, manifestando la disposición a utilizar canales de comunicación emergentes, hecho que puede suponer una tendencia de cambio respecto a la hegemonía del uso de canales tradicionales utilizados por los maestros en ejercicio.

Del mismo modo, se evidencia un cambio en el pensamiento de los maestros en formación en torno al origen de la relación con las familias, mostrándose un tránsito de planteamientos correctivos (diagnósticos de dificultades o necesidades), hacia planteamientos colaborativos donde el conocimiento y comprensión de lo que acontece en el contexto escolar por parte de las familias es fundamental para construir una comunidad educativa participativa. Al respecto, los maestros en formación consideran que la participación de las familias debe formar parte de la actividad del aula y por lo tanto, ha de favorecerse la entrada de las familias en la escuela, aspecto que se potencia haciendo partícipes a las familias de la metodología que se sigue, compartiendo contenidos generalizables en los que las familias puedan aportar su visión y experiencia, acompañando el proceso de aprendizaje de sus hijos e hijas, favoreciendo así una participación continuada en el tiempo.

Finalmente cabe señalar que la presente investigación muestra los resultados de un estudio exploratorio sobre las creencias de los futuros maestros que pretende evidenciar cómo se ven influenciadas por el practicum, que si bien puede tener limitaciones debido a una muestra que podría ser mayor, los datos recogidos y las conclusiones extraídas son relevantes para la comunidad educativa y científica por cuanto permiten acercarse a las creencias de los futuros docentes para evidenciar cuáles son, cómo cambian o se mantienen y cuál es el impacto del practicum o prácticas profesionales educativas en dichas creencias, así como en qué medida desde las aulas universitarias se puede favorecer la reflexión crítica respecto a las mismas.

Futuras líneas de investigación derivadas de este estudio se encaminan hacia la recogida de información de maestros en formación que hayan realizado su período de prácticas profesionales educativas en diferentes tipos de centros educativos, o durante diferentes períodos de tiempo, para poder contrastar el impacto en sus creencias a lo largo de su período formativo. El interés nos llevaría a trabajar con 
maestros en ejercicio que inician su trayectoria profesional, para poder conocer cómo se modifica o cómo se consolida su pensamiento docente, respecto a la relación familia-escuela y a otras temáticas relevantes para mejorar la calidad educativa.

\section{REFERENCIAS}

Alcalay, L.; Milicic, N.; Torretti, A. Alianza efectiva familia-escuela. Un programa audiovisual para padres. Psykhe, Santiago: Pontificia Universidad Católica de Chile, v. 14, n. 2, p. 149-161, 2005.

АматеA, E. Building culturally responsive family-school relationships. Boston, MA: Pearson/Allyn and Bacon, 2009.

Argos, J. La mejora de la educación infantil desde el análisis del pensamiento práctico de sus educadores. International Journal of Early Childhood, Netherlands: Springer, v. 32, n. 2, p. 12-23, 2000.

Arnáiz, V. Los padres en la escuela infantil ¿Clientes o cooperadores? Cuadernos de Pedagogía, Madrid: Wolters Kluwer, n. 282, p. 35-39, 1999.

BASTIANI,J. Working with parents: a whole school approach. Slough: NFER-Nelson, 1989. Batanova, M.; Loukas, A. Unique and interactive effects of empathy, family, and school factors on early adolescents' aggression. Journal of Youth and Adolescence, United Kingdom: Springer, v. 43, n. 11, p. 1.890-1.902, 2014.

Blank, M. A.; Kershaw, C. (Eds.). The design book for building partnerships: school, home and community. Lancaster, PA: Technomic, 1998.

Boult, B. 176 ways to involve parents: practical strategies for partnering with families. Thousand Oaks, CA: Corwin Press, 2006.

Brookmeyer, K.; Fanti, K.; Henrich, C. Schools, parents, and youth violence: a multilevel, ecological analysis. Journal of Clinical Child and Adolescent Psychology, United Kingdom: Taylor \& Francis, n. 35, p. 504-514, 2006.

Castro, A.; García-Ruiz, R. La visión del profesorado de educación infantil y primaria de Cantabria sobre la participación y las relaciones interpersonales entre los miembros de la comunidad escolar. Aula Abierta, Oviedo: Elsevier, v. 41, n. 12, p. 73-84, 2013.

ChAn, K.W.; ElLiott, R. Epistemological beliefs across cultures: critique and analysis of beliefs structure studies. Educational Psychology, United Kingdom: Taylor \& Francis, v. 24, n. 2, p. 123-142, 2004a.

;___ Relational analysis of personal epistemology and conceptions about teaching and learning. Teaching and Teacher Education, United Kingdom: Elsevier, n. 20, p. 817-831, 2004b.

Comisión Europea. Informe Europeo sobre la calidad de la educación escolar. Dieciséis indicadores de calidad. Universidad Nacional de Educación a Distancia; Sociedad Española de Educación Comparada, 2000. Disponible en: <http://www. parentsparticipation.eu/sites/default/files/pagina/6._comision_eu_2000.pdf $\geq$. Acceso en: 2 mar. 2016. 
Consejo Escolar del Estado. La participación de las familias en la educación escolar. Madrid: Ministerio de Educación Ciencia y Deporte, 2014.

Cross, D.; Barnes, A. Using Systems Theory to Understand and Respond to Family Influences on Children's Bullying Behavior: Friendly Schools Friendly Families Program. Theory into Practise, United Kingdom: Taylor \& Francis, v. 53, n. 4, p. 293-299, 2014.

De La Cruz, M.; Pozo, J. I.; Huarte, M.; Scheuer, N. Concepciones de enseñanza y prácticas discursivas en futuros profesores. In: Pozo, J. et al. (Eds.). Nuevas formas de pensar la enseñanza y el aprendizaje. Barcelona: Graó, 2006. p. 359-371.

De Oliveira e Silva, I. A creche e as famílias: o estabelecimento da confiança das mães na Instituição de Educação Infantil1. Educar em Revista, Curitiba: UFPR, n. 55, p. 253-272, 2014.

DiAs, L. R. Formação de professores, educação infantil e diversidade étnico-racial: saberes e fazeres nesse processo. Revista Brasileira de Educação, Rio de Janeiro: ANPEd; Campinas: Autores Associados, v. 17, n. 51, p. 661-749, 2012.

Epstein, J. School, family, community partnerships: caring for the children we share. Phi Delta Kappan, United Kingdom: Sage, v. 79, n. 9, p. 701-712, 1995.

. School, family, and community partnerships. Preparing educators and improving schools. Boulder, CO: Westview Press, 2001.

Eurydice. Una década de reformas en la educación obligatoria de la Unión Europea. Bruselas: Unidad Europea de Eurydice, 1997.

Feito, R. CEAPA y CONCAPA: dos modelos de participación de los padres. Conferencia de Sociología de la Educación, 1., 1992, Madrid.Anais... Madrid: CIDE, 1992.

Fernández-Río, J.; Méndez-Giménez, A. Articulando conocimiento teórico y práctica educativa. Análisis de los efectos del material autoconstruido en las creencias de futuros docentes. Infancia y Aprendizaje, United Kingdom: Taylor \& Francis, v. 36, n. 1, p. 61-75, 2013.

Furman, G. The ethic of community. Journal of Educational Administration, United Kingdom: Sage, v. 42, n. 2, p. 215-235, 2004.

García-Bacete, F. Las relaciones escuela-familia: un reto educativo. Infancia y Aprendizaje, United Kingdom: Taylor \& Francis, v. 26, n. 4, p. 425-437, 2003.

García, M. P.; Gomáriz, M. A.: Hernández-Pradoos, M. A.; Parra, J. La comunicación entre la familia y el centro educativo, desde la percepción de los padres y madres de los alumnos. Educatio Siglo XXI, Murcia, Universidad de Murcia, v. 28, n. 1, p. 157-188, 2010.

Garreta,J. La relación familia-escuela. Lleida: Edicións de la Universitat de Lleida, 2007. . La participación de las familias en la escuela. Las asociaciones de madres y padres de alumnos. Madrid: CIDE/CEAPA, 2008.

La comunicación familia-escuela en educación infantil y primaria. Revista de la Asociación de Sociología de la Educación, Valencia: Universitat de València, n. 1, p. 71-85, 2015. 
Gergen, K. Realidades y relaciones. Aproximaciones a la construcción social. Barcelona: Paidós, 1996.

GoRski, P.What we're teaching tea-chers: an analysis of multicultural teacher education coursework syllabi. Teaching and Teacher Education, United Kingdom: Elsevier, v. 25, n. 2, p. 309-318, 2009.

Grant, K. B.; RAY, J. A. Home, school and community collaboration: culturally responsive family involvement. Los Angeles: Sage, 2010.

Hernández, M. A.; Gomáriz, M. A.; Parra, V.J.; García, M.P. Familia, inmigración y comunicación con el centro escolar: un estudio comparativo. Educación XXI, Madrid: UNED, v. 19, n. 2, p. 127-151, 2016.

Hester, H. Start at home to improve home-school relations. NASSP Bulletin, United Kingdom: Sage, n. 73, p. 23-27, 1989.

KÑallinsky, E. La participación educativa: familia y escuela. Las Palmas: Universidad de Las Palmas de Gran Canarias, 1999.

LAtorre, M. J. El potencial formativo del practicum: cambio en las creencias que sobre la enseñanza práctica poseen los futuros maestros. Revista de Educación, Madrid: Ministerio de Educación, Cultura y Deporte, n. 43, p. 249-273, 2007.

Llevot, N.; Bernard, O. La participación de las familias en la escuela: factores clave. Revista de la Asociación de Sociología de la Educación, Valencia: Universitat de València, v. 8, n. 1, p. 57-70, 2015.

López, I.; Ridao, P.; SÁnchez, J. Las familias y las escuelas: una reflexión acerca de entornos educativos compartidos. Revista de Educación, Madrid: Ministerio de Educación, Cultura y Deporte, n. 334, p. 143-163, 2004.

Luengo, F.; Moya, J. Escuela, familia y comunidad: claves para la acción. Madrid:Wolters Kluwer, 2008.

Marcelo, C. Los comienzos en la docencia: un profesorado con buenos principios. Profesorado - Revista de Curriculum y Formación del Profesorado, Granada: Universidad de Granada, v. 13, n. 1, p. 1-25, 2009.

Marrero, J. (Ed.). El pensamiento reencontrado. Barcelona: Octaedro, 2009.

Martínez, R. et al. Dinamización de las relaciones familia centro escolar a través de la formación del profesorado en este campo de actuación. Revista Española de Orientación y Psicopedagogia, Madrid: UNED, v. 11, n. 19, p. 107-120, 2000.

Martínez,D.J. C.; Ahumada,J. R. C. Teorías subjetivas en profesores y su formación profesional. Revista Brasileira de Educação, Rio de Janeiro: ANPEd; Campinas: Autores Associados, v. 21, n. 65, p. 299-324, 2016. Disponible en: <http://www.scielo.br/scielo. php?script=sci_arttext\&pid=S1413-24782016000200299\&lng=en\&nrm=iso >. Acceso en: 14 abr. 2016.

Maulini, O. La collaboration parents-enseignants dans l'école publique. La Revue des Echanges, Géneve: Université de Géneve, v. 15, n. 4, p. 3-14, 1997. Disponible en: $<$ https://archive-ouverte.unige.ch/unige:35593/ATTACHMENT01>. Acceso en: 26 feb. 2018. 
Navaridas, F.; Raya, E. Indicadores de participación de los padres en el sistema educativo: un nuevo enfoque para la calidad educativa. Revista Española de Educación Comparada, Madrid: UNED, n. 20, p. 223-248, 2012.

Olmsted, P. Parent involvement in elementary education: findings and suggestions from the Follow Through Program. The Elementary School Journal, Chicago: The University of Chicago Press Books, v. 91, n. 3, p. 221-231, 1991.

Pajares, M. F. Teachers'beliefs and educational research: cleaning up a messy construct. Review of Educational Research, United Kingdom: Sage, n. 62, p. 307-332, 1992.

Parreira, M.; Walther, A.; Litau, J. Governance of Educational Trajectories in Europe Access, Coping and Relevance of Education for Young People in European Knowledge Societies in Comparative Perspective. Project Final Report. Frankfurt:University of Frankfurt, 2013. Pozo,J.I. La nueva cultura del aprendizaje en la sociedad del conocimiento. In: Pozo,J.et al. (Eds.). Nuevas formas de pensar la enseñanza y el aprendizaje. Barcelona: Graó, 2006.p.29-54. Prieto, M. Creencias de los profesores sobre evaluación y efectos incidentales. Revista de Pedagogia, Caracas: Universidad de Venezuela, v. 29, n. 84, p. 123-144, 2008.

Santos, M. A. El crisol de la participación. Archidona: Aljibe, 1999.

Tigchelaar, A.; Korthagen, F. Deepening the exchange of student teachingexperiences: Implications for the pedagogy of teacher education of recent insights into teacher behavior. Teaching and Teacher Education, United Kingdom: Elsevier, n. 20, p. 665-679, 2004.

Tirado, R.; Aguaded, I. Influencias de las creencias del profesorado sobre el uso de las tecnologías en el aula. Revista de Educación, Madrid: Ministerio de Educación, Cultura y Deporte, n. 363, p. 230-255, 2014.

\section{SOBRE LOS AUTORES}

Ana Castro Zubizarreta es doctora en psicología por la Universidad de Deusto (España). Profesora de la Universidad de Cantabria (España). E-mail: castroza@unican.es

María Rosa García-Ruiz es doctora en educación por la Universidad Nacional de Educación a Distancia (UNED/España). Profesora de la Universidad de Cantabria (España).

E-mail: rosa.garcia@unican.es

Pablo Maraver López es doctorando en educación por la Universidad de Huelva (España). Profesor de la misma institución. E-mail: pablomaraverlopez@gmail.com 